Pacific Journal of Mathematic 


\title{
SOME CELLULAR SUBSETS OF THE SPHERES
}

\author{
VO-THANH LIEM
}

\begin{abstract}
Let $H$ be a $P L$-homology sphere such that its multisuspension $S^{k} * H$ is topologically homeomorphic to the sphere. We prove that every cell-like subset of $S^{k}$ is cellular. Also, every non-compact $P L$-manifold of dimension greater than four accepts uncountably many simplicial triangulations each of which contains a non-cellular $k$-simplex for every $k \neq 0$.
\end{abstract}

Introduction. The double suspension problem introduces many different simplicial triangulations for a $P L$-manifold; in particular, the case of the sphere. It is easy to see that these strange triangulations are not locally flat, however, we ask whether their simplexes are cellular. A positive answer is given for every cell-like subset of $S^{k}$, where $S^{k}$ is the suspension sphere in $S^{k} * H$ (Theorem 1), and for every cell-like subset of a codimension- 2 simplex which properly meets every nontrivial face of this simplex (Theorem 2). But it is negative for general cases (Theorem 4).

Finally, combining Theorem 4 and Theorem 5, it follows that there are uncountably many noncellular simplicial triangulations for a noncompact $P L$-manifold of dimension greater than four.

The author wishes to thank J. C. Cantrell for his interesting discussions, R. J. Daverman for his enlightening idea in constructing a noncellular simplicial triangulation of a $P L$-manifold and the referee for his suggestions.

Definitions and Notation. Let $\mathbf{R}^{n}$ denote the $n$-dimensional Euclidean space, $S^{n}$ the boundary $\partial \Delta^{n+1}$ of the standard $(n+1)$-simplex $\Delta^{n+1}$

Let $X$ be a compact subset of a metric space $M$. $X$ satisfies the small loops condition $(S L C)$ if $U V(X \subset M)$ and there is a $\delta>0$ such that each $\delta$-loop in $V-X$ is null-homotopic in $U-X$ (see [3]).

For the notion of cellularity refer to Rushing [8] or McMillan [7]. A finite-dimensional compactum $X$ is cell-like if $X$ has the shape of a point (see [6]).

A simplicial triangulation of a space $M$ is a pair $(T, \varphi)$ where $T$ is a simplicial complex and $\varphi$ is a homeomorphism from $|T|$, the underlying space of $T$, onto $M$. Two triangulations $(T, \varphi)$ and $\left(T^{\prime}, \varphi^{\prime}\right)$ are said to be equivalent if there is a $P L$-homeomorphism $\psi:|T| \rightarrow\left|T^{\prime}\right|$ such that $\varphi=\varphi^{\prime} \psi$. (We refer to Hudson [5] for general notions in the PLcategory.) 
A triangulation $(T, \varphi)$ of a manifold $M^{m}$ is said to be locally flat, if $\varphi \mid$ Int $\sigma^{\prime}$ is locally flat for every simplex $\sigma \in T$; i.e. for every $x \in$ Int $\sigma$ there is a neighborhood $U$ of $\varphi(x)$ in $M^{m}$ such that

$$
(U, U \cap \varphi(\sigma)) \stackrel{\text { TOP }}{\approx}\left(\mathbf{R}^{m}, \mathbf{R}^{i}\right) .
$$

If the $(k+1)$-suspension of a non-simply connected $P L$-homology sphere $H^{m}$ is homeomorphic to $S^{m+k+1}$, (e.g. $k \geqq 1, m \geqq 3$ by Edwards [4]), then from the suspension procedure, $S^{m+k+1}$ can be naturally endowed with a simplicial triangulation $T$ that is $P L$-homeomorphic to $S^{k} * H^{m}$, the join of $S^{k}$ and $H^{m}$. Now, if $K$ is any subdivision of $S^{k}$, then $K * H^{m}$ is a subdivision of $S^{k} * H^{m}$. $\quad T$ also denotes this triangulation.

Let $P$ be a subpolyhedron of $S^{k}$. Then there are subdivisions $K$ and $L$ of $S^{k}$ and $P$ respectively such that $L$ is a subcomplex of $K$.

Let $\sigma \in L$ and $\epsilon>0$. Denote

$$
\begin{aligned}
& \stackrel{\circ}{\sigma}=\{x \in \sigma \mid \text { all barycentric coordinates of } x \text { are positive }\} . \\
& \bar{V}(\sigma, \epsilon)=\left\{\begin{array}{l|l}
\alpha x+\beta y+\gamma z & \begin{array}{l}
\alpha, \beta, \gamma \geqq 0, \alpha+\beta+\gamma=1 \\
\alpha+\beta+\gamma=1, \beta+\gamma \leqq \epsilon \\
x \in \sigma, y \in \operatorname{link}(\sigma, K) \\
z \in H
\end{array}
\end{array}\right\} . \\
& V(\sigma, \epsilon)=\{\alpha x+\beta y+\gamma z \in \bar{V}(\sigma, \epsilon) \mid \beta+\gamma<\epsilon\}, \\
& V(\stackrel{\circ}{\sigma}, \epsilon)=\{\alpha x+\beta y+\gamma z \in V(\sigma, \epsilon) \mid x \in \stackrel{\circ}{\sigma}\} .
\end{aligned}
$$

\section{Results and details of proofs.}

LEMma 1. Every proper subpolyhedron of $S^{k} \subset S^{k} * H^{m}$ satisfies the $S L C$ in $S^{k} * H^{m}, k \geqq 1$.

Proof. We will use the notation $K, L, T$ as above. Given a neighborhood $U$ of $L$ in $S^{n}$, there is an $\epsilon>0$ such that $\bar{V}(\sigma, \epsilon) \subset U$ for every $\sigma \in L$.

Let

$$
V=\bigcup_{\sigma \in L} V(\sigma, \epsilon / 2) \subset \bigcup_{\sigma \in L} \bar{V}(\sigma, \epsilon / 2)
$$

Then $V$ is a neighborhood of $L$ in $U$ and the family $\{V(\stackrel{\circ}{\sigma}, \epsilon) \mid \sigma \in L\}$ is an open cover of the compact subset $\cup\{\bar{V}(\sigma, \epsilon / 2) \mid \sigma \in L\}$. 
Let $\delta$ be the Lebesgue number of this covering. Then, we claim that $(V, \delta)$ is a $S L C$ data for $X \subset U$.

Given a loop $f: \partial \Delta^{2} \rightarrow V-L$ of diameter less than $\delta$, we have $f\left(\partial \Delta^{2}\right) \subset V(\stackrel{\circ}{\sigma}, \epsilon)$ for some $\sigma \in L$. By a small homotopy in $V-L$, we can assume that $f\left(\partial \Delta^{2}\right) \cap S^{k}=\varnothing$.

Moreover, every point in $V(\stackrel{\circ}{\sigma}, \epsilon)-\left((\sigma * H) \cup S^{k}\right)$ can be written uniquely in the form $\alpha x+\beta y+\gamma z$ where $\alpha, \beta, \gamma \in(0,1)$ with $\alpha+\beta+$ $\gamma=1, \beta+\gamma<\epsilon$, and $x \in \operatorname{link}(\sigma, K), z \in H$. Hence, if $\Sigma$ denotes $\left\{t \in \partial \Delta^{2} \mid f(t) \notin \sigma * H\right\}$ then $f \mid \Sigma$ can be defined to be

$$
f(t)=\alpha(t) x(t)+\beta(t) y(t)+\gamma(t) z(t)
$$

where $\alpha, \beta, \gamma: \Sigma \rightarrow(0,1)$ with $\beta(t)+\gamma(t)<\epsilon$, and $x, y, z$ are continuous maps from $\Sigma$ to $\sigma, \operatorname{link}(\sigma, K), H$ respectively.

Now, define $F: \partial \Delta^{2} \times I \rightarrow V(\sigma, \epsilon)-L$ by

$$
F(t, s)=\left\{\begin{array}{l}
f(t), \quad \text { if } t \notin \Sigma ; \\
\alpha(t) x(t)+(1-s) \beta(t) y(t)+ \\
(\gamma(t)+s \beta(t)) z(t), \quad \text { if } t \in \Sigma
\end{array}\right.
$$

Then, it can be shown that the restriction of $F$ on each closed subset $f^{-1}(\tau)$ is continuous, where $\tau$ is a $(k+m+1)$-simplex of $S^{k} * H$. Hence, $F$ homotopes $F_{0}=f$ to $F_{1}$ whose image lies in $[(\sigma * H) \cap V(\sigma, \epsilon)]-L$.

By use of the convex structure of the simplex $\sigma$ and the property $0<\gamma(t)<\epsilon$, one then can homotope $F_{1}$ in $V(\sigma, \epsilon)-L$ into a loop, say $f^{\prime}$, in $[(v * H) \cap V(\sigma, \epsilon)]-\{v\}$, where $v$ is a vertex of $\sigma$.

Moreover, since $L$ is a proper subcomplex of $K$, there are vertices $\boldsymbol{w}_{0}=\boldsymbol{v}_{0}, w_{1}, \cdots, w_{q+1}$ of $K$ such that the 1-simplex $\left\langle\boldsymbol{w}_{i-1}, w_{t}\right\rangle \in L$ for $i=1, \cdots, q$ and $\left\langle w_{q}, w_{q+1}\right\rangle \notin L$. Thereby, it is easy to show inductively that $f^{\prime}$ is homotopic in $\left.\left[U \cap\left(\bigcup_{i=1}^{q}\left\langle w_{i-1}, w_{i}\right\rangle * H\right\rangle\right)\right]-L$ to a loop

$$
f^{\prime \prime}: \partial \Delta^{2} \rightarrow\left[V\left(w_{q}, \epsilon\right) \cap w_{q} * H\right]-L .
$$

Denote

$$
f^{\prime \prime}(t)=\alpha_{q}(t) w_{q}+\gamma(t) z(t), \quad(0<\gamma(t)<\epsilon) .
$$

Now, if one defines homotopies $F^{\prime}, F^{\prime \prime}: \partial \Delta^{2} \times I \rightarrow U-L$ by

$$
\begin{aligned}
& F^{\prime}(t, s)=\left(\alpha_{q}(t)-s \epsilon / 2\right) w_{q}+s \epsilon w_{q+1} / 2+\gamma(t) z(t), \\
& F^{\prime \prime}(t, s)=\left(\alpha_{q}(t)-\epsilon / 2+s \gamma(t)\right) w_{q}+\epsilon w_{q+1} / 2+(1-s) \gamma(t) z(t)
\end{aligned}
$$

then, the glueing of $F^{\prime}$ and $F^{\prime \prime}$ homotopes $f^{\prime \prime}$ to a constant map in $U-L$. Thus, the lemma is proved. 
THEOREM 1. If $S^{k} * H^{m}$ is homeomorphic to $S^{k+m+1}$, then every cell-like subset $X$ of $S^{k} \subset S^{k} * H^{m}$ is cellular in $S^{n}$. In particular, every simplex of $S^{k}$ is cellular in $S^{n}$.

Proof. By [3], it suffices to show that $X$ satisfies the SLC (or $X$ satisfies the cellularity criterion, then apply the main theorem of [7]), since $X$ has the shape of a point.

Given an open neighborhood $U$ of $X$ in $S^{n}$. Let $K$ be such a fine triangulation of $S^{k}$ that

$$
L=\cup\{\sigma \in K \mid \sigma \cap X \neq \varnothing\} \subset U \cap S^{k} .
$$

Furthermore, using a regular neighborhood argument, we may assume that $L$ is a neighborhood of $X$ in $S^{k}$.

By Lemma 1, it follows that there is a neighborhood $V$ of $L$ in $U$ (also a neighborhood of $X$ in $U$ ) and $\delta>0$ such that every $\delta$-loop in $V-L$ is null-homotopic in $U-L$. We claim that $(V, \delta / 2)$ is a $S L C$ data for $X$ in $U$.

Let $f: \partial \Delta^{2} \rightarrow V-X$ such that $\operatorname{diam} f\left(\partial \Delta^{2}\right)<\delta / 2$. Since $L$ is a codimension-4 compact subset of $V(P L$-manifold $), V-L$ is 0 $U L C$. Thus, by use of a fine triangulation of $\partial \Delta^{2}$ we can redefine $f$ skeletonwise to obtain a new map $f^{\prime}$ such that

(i) $f^{\prime}\left(\partial \Delta^{2}\right) \subset V-L$,

(ii) $\operatorname{diam} f^{\prime}\left(\partial \Delta^{2}\right)<\delta$,

(iii) $f$ is homotopic to $f^{\prime}$ in $V-X \subset U-X$.

The claim follows by homotoping $f^{\prime}$ to a constant map in $U-L \subset$ $U-X$. Then, Theorem follows.

It is interesting to point out the following corollary.

COROLlARY. If $H^{n}$ is a homology $n$-sphere $(n \geqq 3)$ and for $n=3$, $H^{3}$ bounds a contractible topological 4-manifold, then every subarc of $S^{1}$ in $S^{1} * H^{n}$ is cellular.

We omit the standard proof of the following sublemma.

Sublemma. Given a codimension-2 cell-like subset $X$ of the sphere $S^{n}$, then $X$ is globally 1-alg (refer to [9]) if it satisfies the SLC.

A compact subset $X$ of a manifold $M$ properly intersects a simplex $\sigma$ of a simplicial triangulation $K$ of $M$ if $X \cap|\sigma| \neq|\sigma|$.

THEOREM 2. Given a cell-like subset $X$ of an $(n-2)$-simplex $\sigma$ of a simplicial triangulation $K$ of a PL-manifold $M^{n}$ ( $\left.n \geqq 5\right)$, if $X$ properly intersects every nontrivial face (not a vertex) of $\sigma$, then it is cellular in $M$. 
In particular, every proper subarc of a 1-simplex is cellular.

Proof. By Theorem 1 of [9], it suffices to show that $X$ is globally 1-alg (or $X$ satisfies the cellularity criterion in order to apply the main theorem of [7]), since $X$ has the shape of a point. However, by Sublemma it suffices to show that $X$ satisfies the $S L C$.

Similar to the proof of Theorem 1, it suffices to show that $X$ has arbitrarily small $P L$-manifold neighborhood $L$ 's in $\sigma^{n-2}$ which satisfy the $S L C$ (i.e. the conclusion of Lemma 1).

Let $K^{\prime}$ be a triangulation of $M$ such that $\sigma^{\prime} * \operatorname{link}(\sigma, K)$ is a subcomplex, where $\sigma^{\prime}$ is the fine subdivision of $\sigma$ used to define the small neighborhood $L$ of $X$ in $\sigma$. Since $X$ intersects every face of $\sigma$ properly, we may assume $L$ does also.

Similar to Lemma 1, given a neighborhood $U$ of $L$, there correspond a neighborhood $V$ of $L$ in $U$ and $\delta>0$, a Lebesgue number of the open cover $\{V(\stackrel{\circ}{\sigma}, \epsilon) \mid \sigma \in L\}$ less than $\frac{1}{2} \operatorname{mesh}(L)$ (refer to [8]).

Given a loop $f: \partial \Delta^{2} \rightarrow V-L$, we have the following cases.

Case 1. $f\left(\partial \Delta^{2}\right) \subset V(v, \epsilon)$ for some vertex $v$ of $L$ lying in $\stackrel{\circ}{\sigma}$.

Since $\stackrel{\circ}{\sigma}$ is locally flat and codimension-2, we may assume that $f\left(\partial \Delta^{2}\right) \cap \sigma=\varnothing$ by a small homotopy in $V-L$. Hence, $f(t)$ can be written uniquely in the form

$$
f(t)=\alpha(t) x(t)+\beta(t) y(t) \quad\left(t \in \partial \Delta^{2}\right),
$$

where $x(t) \in \stackrel{\circ}{\sigma}$ near $v, y(t) \in|\operatorname{link}(\sigma, K)|$ etc ... as in Lemma 1 .

Then, we can homotope $f$ into $|v * \operatorname{link}(\sigma, K)|-|\sigma|$. As in Lemma $1, f$ is homotopic to $f^{\prime}$ such that $f^{\prime}\left(\partial \Delta^{2}\right) \subset\left|w_{q} * \operatorname{link}(\sigma, K)\right|-|\sigma|$, where $w_{q}$ is a vertex of $L$ in $\stackrel{\circ}{\sigma}$ such that $\operatorname{star}\left(w_{q}, \sigma^{\prime}\right) \not \subset L$. Finally, by a small homotopy we can move $f^{\prime}$ into $\left|\left(\epsilon w_{q+1} / 2+(2-\epsilon) w_{q} / 2\right) * \operatorname{link}(\sigma, K)\right|$ and shrink it in there. This completes the proof of the Case 1.

Case 2. $f\left(\partial \Delta^{2}\right) \subset V(\stackrel{\circ}{\tau}, \epsilon)$, where $\tau$ is a simplex of $L$ such that $\stackrel{\circ}{\tau} \stackrel{\circ}{\sigma}$.

As in the Case 1, we may assume that $f\left(\partial \Delta^{2}\right) \cap \sigma=\varnothing$. Hence, $f(t)$ can be written in the form $(*)$.

Now, the join structure and a homotopy in $\stackrel{\circ}{\tau}$ moving $x(t)$ close to a vertex $v$ of $\tau(v \in \stackrel{\circ}{\sigma})$ provide a homotopy in $V-\sigma \subset V-L$ that moves $f$ into $V(v, \epsilon)-\sigma$. Then, case 1 will complete the shrinking.

Case 3. $f\left(\partial \Delta^{2}\right) \subset V(\tau, \epsilon)$, where $\tau$ is a simplex of $L$ and $\stackrel{\tau}{\tau} \subset \sigma_{1}$ with $\sigma_{1}$ being a nontrivial proper face of $\sigma$. 
Extend $f$ to $\bar{f}: \Delta^{2} \rightarrow V(\stackrel{\circ}{\tau}, \epsilon)$.

Since $V(\stackrel{\circ}{\tau}, \epsilon)-|\sigma|$ is $0-U L C(\operatorname{dim} \sigma=n-2)$, by use of a fine subdivision $J$ of $\Delta^{2}$ we can redefine skeletonwise $\bar{f} \mid J^{(1)}$ to obtain a map $f^{\prime}$ on the 1-skeleton $J^{(1)}$ of $J$ such that

(i) $f^{\prime}: J^{(1)} \rightarrow V(\stackrel{\circ}{\tau}, \epsilon)-|\sigma|$,

(ii) $\bar{f} \mid J^{(1)}$ is homotopic to $f^{\prime}$ in $V(\stackrel{\circ}{\tau}, \epsilon)-L$.

Therefore, without loss of generality we may assume that $f\left(\partial \Delta^{2}\right) \cap$ $|\sigma|=\varnothing$. Then, the equation $(*)$ can be used for this case with $y(t) \in$ $\mid$ link $\left(\sigma_{1}, K\right)|-| \sigma_{2} \mid$, where $\sigma_{2} \in K$ such that $\sigma=\sigma_{1} * \sigma_{2}$.

Combining appropriately the proof of Cases 1 and 2 to homotope $x(t)$, it follows that $f$ is homotopic in $V-L$ to a loop $f^{\prime \prime}$ in $\left|w *\left(\left|\operatorname{link}\left(\sigma_{1}, K\right)\right|-\left|\sigma_{2}\right|\right)\right|$ where $w$ is a vertex of $L$ in ${\stackrel{\circ}{\sigma_{1}}}_{\text {. }}$ Then, the last step in Case 1 completes the proof.

Case 4. $f\left(\partial \Delta^{2}\right) \subset V(v, \epsilon)$, where $v$ is a vertex of $\sigma$.

Extend $f$ to $\bar{f}: \Delta^{2} \rightarrow V(v, \epsilon)$. We assume that $\bar{f}\left(\Delta^{2}\right)$ misses an open neighborhood of $v$; hence, $\bar{f}^{-1}(L-\{v\})$ is a compact subset of Int $\Delta^{2}$ and it is covered by the family $\{V(\tau, \epsilon) \mid \tau \in L$ with $v$ as a vertex $\}$.

By use of a fine triangulation of $\Delta^{2}$, we can obtain a compact $P L$-manifold $N$ of $\bar{f}^{-1}(L-\{v\})$ such that $N$ is carried into the union of the family $\{V(\stackrel{\circ}{\tau}, \epsilon) \mid \tau \in L$ with $v$ as a vertex $\}$.

Now, we subdivide $N$ so fine that the diameter of $\bar{f}(\eta)$ is less than the Lebesgue number of the covering for every 2-simplex $\eta$ of this new subdivision $N_{1}$.

Again, we can redefine $\bar{f}$ on the 1 -skeleton $N_{1}^{(1)}$ of $N_{1}$ to obtain a map $f^{\prime}$ such that

(i) $f^{\prime}: \partial \eta \rightarrow V(\stackrel{\circ}{\tau}, \epsilon)$, for some $\tau$,

(ii) $f^{\prime}|\partial N=\bar{f}| \partial N$,

(iii) $\bar{f} \mid N_{1}^{(1)}$ is homotopic to $f^{\prime}$ in $V-L$.

Hence, the problem is reduced to Case 1,2 or 3 .

The proof of Theorem 2 is complete.

Remark. It should be mentioned that the condition that $X$ properly meet every nontrivial face of $\sigma$ seems to be essential by Theorem 4 below.

LEMMA 2. Let $\sigma=\sigma_{1} * \sigma_{2}$ be a simplex of $T$, where $\sigma_{1}, \sigma_{2}$ are simplexes of $K$ and $H$ respectively. Then, $|\sigma|-\left|\sigma_{1}\right|$ is locally flat in $S^{n}$.

Lemma 3. Let $D_{1}$ be a PL-ball in $S^{k}$ and $D_{2}$ a PL-ball in $H^{m}$. Then, $D_{1} * D_{2}-D_{1}$ is locally flat in $S^{n}$.

Proof. Lemma follows by use of the join structure, the unknottedness of $D_{1}$ and $D_{2}$ in $S^{k}$ and $H$ and Lemma 2. 
Before stating the consequence of this Lemma, the notion of pseudo-flatness should be defined.

Definition. Let $D$ be a $d$-cell in $\mathbf{R}^{n}$ (or $S^{n}$ ), $X$ a subset of $D$ such that $D-X$ is locally flat in $\mathbf{R}^{n}$ (or $S^{n}$ ). The pair $(D, X)$ is said to be pseudo-flat in $\mathbf{R}^{n}$ if given a homeomorphism $g$ from $D$ onto the standard $d$-ball $B$, there is a function $G$ extending $g$ over $\mathbf{R}^{n}$ onto itself such that

$$
G \mid \mathbf{R}^{n}-X: \mathbf{R}^{n}-X \rightarrow \mathbf{R}^{n}-g(X)
$$

is a homeomorphism.

Theorem 3. Given PL-balls $D_{1}$ and $D_{2}$ of $S^{k}$ and $H$ respectively, then the pair $\left(D_{1} * D_{2}, D_{1}\right)$ is pseudo flat in $\mathbf{R}^{n}=\left(S^{n}-p t\right)$.

Proof. From Lemma 2, it follows that $D_{1} * D_{2}-D_{1}$ is locally flat in $S^{n}$. It is clear that $D_{1}$ is cellular $D_{1} * D_{2}$; thus, since $D_{1}$ is cellular in $S^{n}$ by Theorem 1, the proof of Theorem 3 is completed by Cantrell and Lacher's theorem [2].

Let us introduce some convenient notation before proceeding to construct noncellular triangulations of a $P L$-manifold.

Let $\bigcup_{t=1}^{n}\left\langle a_{t}, b\right\rangle * L$ be a subcomplex of a simplicial complex $K$ and

$$
\varphi: \bigcup_{i=1}^{n}\left\langle a_{t}, b\right\rangle * L \rightarrow b * L
$$

the linear map determined by

(i) $\varphi=i d$. on $b * L$, and

(ii) $\varphi\left(a_{t}\right)=b(i=1, \cdots, n)$.

A decomposition $G$ of $|K|$ is said to be associated with $\varphi$ if each of its nondegenerate elements is the set $\varphi^{-1}(x)$ for some $x \in b * L$.

Let $A$ be a $G$-saturated subset of $|K|$. Denote $G_{A}, G \mid A$ the decompositions of $|K|, A$ respectively, whose nondegenerate elements are those of $G$ lying in $A$.

LEMMA 4. Every $n$-sphere $(n \geqq 5)$ accepts a simplicial triangulation having a noncellular 1-simplex.

Proof. Let $v$ be a vertex of a $P L$-homology $(n-2)$-sphere $H$ such that $H * S^{1} \approx S^{n}$. Denote $w_{1}, w_{1}^{\prime}, w_{2}, w_{2}^{\prime}$ the vertices of the suspension sphere $S^{1}=S_{1}^{0} * S_{2}^{0}$. The outline of the proof is as follows.

Consider the 2-cell $v * S^{1}$ whose complement in $S^{n}$ is not simply connected. We will construct a decomposition $G$ of $S^{n}$ whose quotient space $S^{n} / G$ has a simplicial triangulation with $v * S^{1} /\left(G \mid v * S^{1}\right)$ as a non-cellular 1-simplex, and $S^{n} / G \approx S^{n}$. 
To do that, first consider the decomposition $G_{1}$ of $S^{n} \approx H * S^{1}$ associated with the linear map

$$
\varphi: S_{1}^{0} * v * S_{2}^{0} \rightarrow v * S_{2}^{0}
$$

defined by

(i) $\varphi=i d$. on $v * S_{2}^{0}$,

(ii) $\varphi\left(S_{1}^{0}\right)=v$.

Then, Fact 1 below shows that $S^{n} / G_{1} \approx S^{n}$, and it is easy to show that $S^{n} / G_{1}$ induces from $T$ a simplicial triangulation $T_{1}$ of $S^{n}$ with $\tilde{v} * \tilde{S}_{2}^{0}$ as a subcomplex, where $\tilde{v}=\left\{v * S_{1}^{0}\right\}$ and $\tilde{S}_{2}^{0} \equiv S_{2}^{0}$.

Then, by Fact 2 below $\left\langle\tilde{v}, \tilde{w}_{2}\right\rangle$ is cellular in $S^{n} / G_{1}$. Hence, we have

$$
S^{n} /\left\langle\tilde{v}, \tilde{w}_{2}\right\rangle \approx S^{n}
$$

(Corollary 1.8.1, [8].)

Therefore, we can obtain naturally from $T_{1}$ a desired simplicial triangulation of $S^{n}$.

Now, we will complete the proof of Lemma 4 by proving Facts 1 and 2 .

Fact 1. $\left(H * S^{1}\right) / G_{1} \approx S^{n}$.

Consider the $\operatorname{arc} \alpha=v * S_{1}^{0}$ in $H * S_{1}^{0}$. It is clear that $\left(H * S^{1}\right) / G_{1}=$ $\left(\left(H * S_{1}^{0}\right) / \alpha\right) * S_{2}^{0}$.

Hence, it suffices to show that

$$
\left(\left(H * S_{1}^{0}\right) / \alpha\right) \times \mathbf{R} \approx\left(H * S_{1}^{0}\right) \times \mathbf{R} .
$$

However, the proof of Andrews and Curtis' theorem $\left(\left(\mathbf{R}^{n} / \alpha\right) \times \mathbf{R} \approx\right.$ $\mathbf{R}^{n+1}$ ) also works if we replace $\mathbf{R}^{n}$ by $H^{n} * S^{0}$ (see Chapter 2.5 of [8]). So, Fact 1 follows.

Fact 2. $\left\langle\tilde{v}, \tilde{w}_{2}\right\rangle$ is cellular in $S^{n} / G_{1} \approx S^{n}$.

It is easy to see that every homeomorphism of $v * S^{1}$ onto itself induces from the join structure a homeomorphism of $S^{n} \approx H * S^{1}$ onto itself.

Now, consider a homeomorphism $h$ of $v * S^{1}$ such that

(i) $h \circ \varphi=\varphi \circ h$

(ii) $h\left(\left\langle v, w_{2}\right\rangle\right)$ is the proper subarc $\left\langle h(v), w_{2}\right\rangle$ of $\left\langle v, w_{2}\right\rangle$, where $\varphi$ is the linear map defining $G_{1}$.

Since such a homeomorphism of $v * S^{1}$ induces a homeomorphism of $S^{n} / G_{1}$ which carries $\left\langle\tilde{v}, \tilde{w}_{2}\right\rangle$ onto the proper subarc $\left\langle h(\tilde{v}), \tilde{w}_{2}\right\rangle$ being cellular in $S^{n}$ by Theorem 2 , it follows trivially that $\left\langle\tilde{v}, \tilde{w}_{2}\right\rangle$ is cellular as desired.

The proof of Lemma 4 is complete. 
LEMMA 5. For each $k(2 \leqq k \leqq n)$, there is a simplicial triangulation of $S^{n}(n \geqq 5)$ with a noncellular $k$-simplex.

Proof. For a fixed $n \geqq 5$, since the ideas of the proof for all cases are exactly the same, we only prove in details for $k=2$ and then will point out necessary modifications for other cases.

Consider $v * S^{1}=v * S_{1}^{0} * S_{2}^{0}$. We will define a decomposition $G$ of $S^{n} \approx H^{n-2} * S^{1}$ which induces from $T$ a simplicial triangulation of $S^{n} / G \approx$ $S^{n}$ such that $\left(v * S^{1}\right) /\left(G \mid v * S^{1}\right)$ is a noncellular 2-simplex.

Step 1. Let $G_{1}$ be the decomposition of $S^{n}$ associated with the linear map $\varphi: v * w_{1} * S_{2}^{0} \rightarrow w_{1} * S_{2}^{0}$ defined by

(i) $\varphi=$ identity on $w_{1} * S_{2}^{0}$,

(ii) $\varphi(v)=w_{1}$.

Then, it is easy to see that there is a pseudo isotopy (refer to [8]) starting at the identity of the ball $\left|v * S^{1} * \operatorname{link}(v, H)\right|$ and ending up with a natural surjective map $\mu$ whose point-inverse images are the elements of $G_{1}$, and $\mu=$ identity on $\partial\left(v * S^{1} * \operatorname{link}(v, H)\right)=\partial\left(H_{+} * S^{1}\right)$, where $H_{+}$ is the closure of $(H-\operatorname{star}(v, H))$ in $H$.

Hence,

$$
\begin{aligned}
S^{n} / G & =\left(H_{+} * S^{1}\right) \cup\left(\operatorname{star}(v, H) * S^{1}\right) / G_{1} \\
& =\left(H_{+} * S^{1}\right) \cup \operatorname{star}(v, H) * S^{1} \\
& \approx S^{n}
\end{aligned}
$$

This decomposition induces naturally from $T$ a simplicial triangulation $T_{1}$ of $S^{n}$ which has only one new vertex in the interior of each 1-simplex $\left\langle\tilde{w}_{1}, b\right\rangle$, where $b$ is a vertex of $\operatorname{link}(v, H)$ and $\tilde{w}_{1}=\mu\left(w_{1}\right)$.

Step 2. We now think of $S^{n}$ as $S^{n} / G_{1}$ endowed with this new triangulation $T_{1}$. Then, $\mu\left(v * S^{1}\right)$ is a subcomplex of $S^{n}$ consisting of two 2-simplexes.

Let $G_{2}$ be the decomposition of $S^{n}$ associated with the collapsing $\operatorname{map} \varphi:\left\langle\tilde{w}_{1}, \tilde{w}_{1}^{\prime}, \tilde{w}_{2}\right\rangle \rightarrow\left\langle\tilde{w}_{1}^{\prime}, \tilde{w}_{2}\right\rangle$ where $\tilde{w}$ 's are $\mu(w)$ 's. We want to claim that $S^{n} / G_{2} \approx S^{n}$; hence, it induces from $T_{1}$ a desired simplicial triangulation of $S^{n}$.

In fact, a similar proof as in Lemma 4 shows that $\left\langle\tilde{w}_{1}, \tilde{w}_{2}\right\rangle$ is cellular in $S^{n}$. Therefore, $S^{n} /\left\langle\tilde{w}_{1}, \tilde{w}_{2}\right\rangle \approx S^{n}$ (Corollary 1.8.1, [8]).

Now, the shrinking corresponding to the rest of $G_{2}$ only happens in the ball $\left(\left|\operatorname{star}(v, H) * S^{1}\right| / G_{1}\right) /\left\langle\tilde{w}_{1}, \tilde{w}_{2}\right\rangle$ and keeps its boundary fixed. Hence, a similar proof as in step 1 shows that $S^{n} / G_{2} \approx S^{n}$.

Finally, this decomposition also induces a simplicial triangulation of $S^{n}$ with $\left(v * S^{1}\right) /\left(G_{2} \mid v * S^{1}\right)$ being a noncellular 2-simplex. This completes the proof for $k=2$. 
For $k>2$, we consider a simplex $v * \tau$ of $H$. The first decomposition $G_{1}$ will be the one associated with the collapsing map $\varphi_{1}: v * w_{1} * S_{2}^{0} * \tau \rightarrow w_{1} * S_{2}^{0} * \tau$ carrying $v$ onto $w_{1}$. Then, also $S^{n} / G_{1} \approx$ $S^{n}$.

Now, the collapsing map $\varphi_{2}:\left\langle\tilde{w}_{1}^{\prime}, \tilde{w}_{1}, \tilde{w}_{2}\right\rangle * \tau \rightarrow\left\langle\tilde{w}_{1}^{\prime}, \tilde{w}_{2}\right\rangle * \tau$ carrying $\tilde{w}_{1}$ to $\tilde{w}_{2}$ defines the second decomposition $G_{2}$. Then, we divide the shrinking process defining by $G_{2}$ into 3 steps:

(a) $S^{n} /\left\langle\tilde{w}_{1}, \tilde{w}_{2}\right\rangle \approx S^{n}$ (as above).

(b) $S^{n} /\left(G_{2}\right)_{\left\langle\tilde{w}_{1}, \tilde{w}_{2}\right\rangle * \tau \mid} \approx S^{n}$.

Since the ball $\left(\left\langle\tilde{w}_{1}, \tilde{w}_{2}\right\rangle * \tau\right) /\left\langle\tilde{w}_{1}, \tilde{w}_{2}\right\rangle$ is locally flat, possibly except at $\left\{\left\langle\tilde{w}_{1}, \tilde{w}_{2}\right\rangle\right\}$, in $S^{n} /\left\langle\tilde{w}_{1}, \tilde{w}_{2}\right\rangle$, it is flat (Corollary 3.4.2, in [8]). Hence, using Theorem 5 of [1], it follows that

$$
S^{n} /\left(G_{2}\right)_{\left\langle\left\langle\bar{w}_{1}, \bar{w}_{2}\right\rangle * \tau\right|} \approx S^{n}
$$

(c) The rest of the shrinking process determined by $G_{2}$ only happens in the ball

$$
\left(\left|\operatorname{star}(v * \tau, H) * S^{1}\right|\left(G_{1}\right) /\left(G_{2}\right)_{\left\langle\tilde{w}_{1}, \tilde{w}_{2}\right\rangle * \tau \mid}\right.
$$

and keeps its boundary fixed. So, we can prove $S^{n} / G_{2} \approx S^{n}$ and a desired simplicial triangulation of $S^{n}$ can be obtained.

The proof of Lemma 5 is complete.

TheOREM 4. Every PL-manifold of dimension greater than 4 accepts a simplicial triangulation that contains a noncellular $k$-simplex for every $k \neq 0$.

Proof. Lemmas 4 and 5 provide a family $\left\{S_{k}^{n} \mid k=1,2, \cdots, n\right\}$ of simplicial triangulations of $S^{n}$ such that each $S_{k}^{n}$ contains a non-cellular $k$-simplex. Then, for every $P L n$-manifold $M$, we have

$$
M \approx M \# S_{1}^{n} \# \cdots \# S_{n}^{n}
$$

where the connected sum happens only in the interior of an $n$-simplex of $S_{k \text {. }}^{n} \quad$ It is clear that we can obtain naturally a desired simplicial triangulation of $M$.

According to the referee's suggestion, it is interesting to include the proof of the following result that appears in the original version as an observation. Basically, the ideas of our proofs are the same.

THEOREM 5. Every noncompact PL-manifold $M^{m}(m \geqq 5)$ accepts uncountably many simplicial triangulations. 
Proof. We consider a countable collection of PL homology sphere $\left\{H_{1}^{m-2}\right\}(m \geqq 3)$, such that

(i) $\pi_{1}\left(H_{i}\right) \neq \pi_{1}\left(H_{j}\right)$ for $i \neq j$, and

(ii) $S^{1} * H_{\imath} \approx S_{\imath}^{m}$.

(Such sequences exist by Glaser and Edwards.) Hence, it induces a sequence of simplicial triangulations $\left\{T_{i}\right\}$ of $\left\{S_{i}^{m}\right\}$ such that every simplex of each $T_{i}$ is locally flat, except the suspension circle $S_{i}^{1}$ (the link of each 1-simplex of $S_{\imath}^{1}$ is $H_{i}$ ).

Now, we enumerate the $m$-simplexes of a $P L$ triangulation of $M$, say $\left\{\sigma_{k}^{m}\right\}_{k=1,2, \cdots}$. To each infinite sequence $\left\{i_{k}\right\}$ we assign a simplicial triangulation of $M$ induced naturally from $M \approx M \# S_{t_{1}}^{m} \# S_{t_{2}}^{m} \# \cdots$, where the connected sum is defined in the interior of $\sigma_{k}^{m}$ and that of an $m$-simplex of $S_{i_{k}}^{m}$ for each $k=1,2, \cdots$.

These triangulations will be $P L$ except for the suspension circles.

To see that the triangulations are different, consider a $P L$ homeomorphism $h$ from $M \# S_{i_{1}}^{m} \# S_{i_{2}}^{m} \# \cdots$ to $M \# S_{i_{1}^{\prime}}^{m} \# S_{i_{2}}^{m} \# \cdots$.

Since the triangulations are $P L$ except on the various suspension circles, the various circles must be carried onto each other. But then, since some $H_{\lambda}$ occurs more in one sequence than the other, the link of a 1-simplex in $S^{1}$ corresponding to this extra $H_{\lambda}$ will be $H_{\lambda}$, and the link of this 1-simplex under $h$ (or $h^{-1}$ ) will not be homeomorphic to $H_{\lambda}$. Since there are uncountably many infinite sequences of positive integers, this contradiction completes the proof.

\section{REFERENCES}

1. R. H. Bing, Upper semicontinuous decompositions of $E^{3}$. Ann. of Math., 65 (1957), 363-374.

2. J. C. Cantrell, Some non-continuous functions and pseudoflatness, Lecture note in Math., Springer-Verlag, 438 (1974), 119-121.

3. D. Coram, R. J. Daverman and P. Duvall, Jr., A small loops condition for embedded compacta, Proc. Amer. Math. Soc., 53 (1975), 205-212.

4. R. D. Edwards,

5. J. F. P. Hudson, Piecewise Linear Topology, W. A. Benjamin, 1969.

6. R. C. Lacher, Cell-like mappings and their generalizations (to appear).

7. D. R. McMillan, A criterion for cellularity in a manifold, Ann. of Math., 79 (1964), 327-337.

8. T. B. Rushing, Topological Embeddings, Academic Press, 1973.

9. Vo-Thanh Liem, Certain continua in $S^{n}$ of the same shape have homeomorphic complements, Trans. Amer. Math. Soc., 218 (1976), 207-217.

Received July 14, 1976 and in revised form November 23, 1976. The author is receiving a postdoctoral fellowship at University of Georgia.

UNIVERSITY OF GEORGIA

Current Address: Louisiana State University

Baton Rouge, LA. 70803 




\section{Pacific Journal of Mathematics}

Vol. 68, No. 1

March, 1977

Richard Julian Bagby, On $L^{p}, L^{q}$ multipliers of Fourier transforms . .......

Robert Beauwens and Jean-Jacques Van Binnebeek, Convergence theorems in

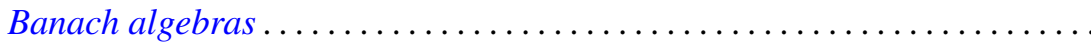

James Cyril Becker, Skew linear vector fields on spheres in the stable

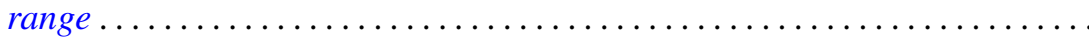

Michael James Beeson, Continuity and comprehension in intuitionistic formal

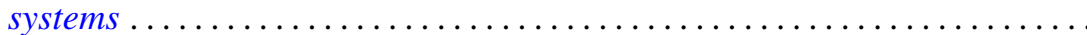

James K. Deveney, Generalized primitive elements for transcendental field

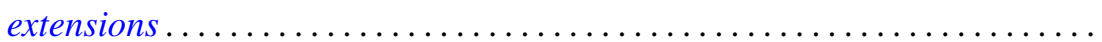

Samuel S. Feder, Samuel Carlos Gitler and K. Y. Lam, Composition properties

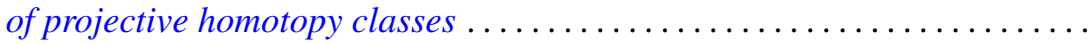

Nathan Jacob Fine, Tensor products of function rings under composition ......

Benno Fuchssteiner, Iterations and fixpoints . . . . . . . . . . . . . .

Wolfgang H. Heil, On punctured balls in manifolds

Shigeru Itoh, A random fixed point theorem for a multivalued contraction

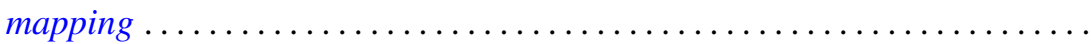

Nicolas P. Jewell, Continuity of module and higher derivations . . . . . . ......

Roger Dale Konyndyk, Residually central wreath products . . . . . . . . . . .

Linda M. Lesniak and John A. Roberts, On Ramsey theory and graphical

parameters.

Vo Thanh Liem, Some cellular subsets of the spheres.

Dieter Lutz, A perturbation theorem for spectral operators

P. H. Maserick, Moments of measures on convex bodies ... . . .

Stephen Joseph McAdam, Unmixed 2-dimensional local domains . .

D. B. McAlister and Norman R. Reilly, E-unitary covers for inverse semigroups...

William H. Meeks, III and Julie Patrusky, Representing codimension-one

homology classes by embedded submanifolds . . .

Premalata Mohapatro, Generalised quasi-Nörlund summability . .

Takahiko Nakazi, Superalgebras of weak-*Dirichlet algebras .

Catherine Louise Olsen, Norms of compact perturbations of operators .

William Henry Ruckle, Absolutely divergent series and isomorphism of

subspaces. II.

Bernard Russo, On the Hausdorff-Young theorem for integral operators .

Arthur Argyle Sagle and J. R. Schumi, Anti-commutative algebras and

homogeneous spaces with multiplications ............

Robert Evert Stong, Stiefel-Whitney classes of manifolds .

D. Suryanarayana, On a theorem of Apostol concerning Möbius functions of

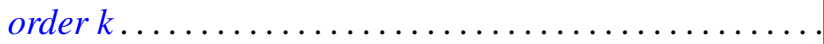

Yoshio Tanaka, On closedness of $C$ - and $C^{*}$-embeddings . . 